\title{
Ras and the Plasma Membrane: A Complicated Relationship
}

\author{
Yong Zhou, Priyanka Prakash, Alemayehu A. Gorfe, and John F. Hancock \\ Department of Integrative Biology and Pharmacology, University of Texas Health Science Center at Houston, \\ Houston, Texas 77030 \\ Correspondence: john.f.hancock@uth.tmc.edu
}

The primary site of Ras signal transduction is the plasma membrane (PM). On the PM, the ubiquitously expressed Ras isoforms, $\mathrm{H}-, \mathrm{N}-$, and $\mathrm{K}$-Ras, spatially segregate to nonoverlapping nanometer-sized domains, called nanoclusters, with further lateral segregation into nonoverlapping guanosine triphosphate (GTP)-bound and guanosine diphosphate (GDP)-bound nanoclusters. Effector binding and activation is restricted to GTP nanoclusters, rendering the underlying assembly mechanism essential to Ras signaling. Ras nanoclusters have distinct lipid compositions as a result of lipid-sorting specificity encoded in each Ras carboxy-terminal membrane anchor. The role of the G-domain in regulating anchor-membrane interactions is becoming clearer. Ras G-domains undergo significant conformational orientation changes on guanine nucleotide switch, leading to differential direct contacts between the Gdomain and reorganization of the membrane anchor. Ras G-domains also contain weak dimer interfaces, resulting in homodimerization, which is an obligate step of nanoclustering. Modulating the formation of Ras dimers, the lipid composition of the PM or lateral dynamics of key PM phospholipids represent novel mechanisms whereby the extent of Ras nanoclustering can be regulated to tune the gain in Ras signaling circuits.

In this review, we will discuss the spatiotemporal organization of Ras proteins on the cell plasma membrane (PM) and the emergent signaling consequences of the attendant spatial distribution of Ras signaling complexes. Specifically, we will discuss how the posttranslationally modified carboxy-terminal anchoring domains of Ras isoforms selectively sort PM lipids and drive isoform-specific nanoclustering. We will then explore the latest findings on G-domain-mediated Ras homodimerization and how Ras dimers potentially contribute to the formation of higher order oligomers. We will conclude the review by discussing the potential biological implications of Ras nanoclusters and how altering lipid composition within Ras nanoclusters influences signal transduction.

\section{RAS ANCHORS AND PLASMA MEMBRANE TARGETING}

Ras superfamily proteins oscillate between inactive guanosine diphosphate (GDP)-bound and active guanosine triphosphate (GTP)-bound states to control signaling cascades that regulate multiple aspects of cell biology, including cell

Editors: Linda VanAelst, Julian Downward, and Frank McCormick

Additional Perspectives on Ras and Cancer in the 21st Century available at www.perspectivesinmedicine.org

Copyright (C) 2018 Cold Spring Harbor Laboratory Press; all rights reserved; doi: 10.1101/cshperspect.a031831

Cite this article as Cold Spring Harb Perspect Med 2018;8:a031831 
Y. Zhou et al.

survival, migration, and proliferation. Prototypical examples are the three Ras isoforms that are ubiquitously expressed in human cells: $\mathrm{K}-, \mathrm{H}$-, and N-Ras, of which K-Ras and N-Ras are frequently mutated in human cancers (Prior et al. 2012). Ras proteins contain nearly identical G-domains that bind guanine nucleotides and interact with a common set of activators and effectors but show significant sequence variation in their carboxy-terminal hypervariable regions (Prior and Hancock 2001; Hancock 2003). For biological activity, Ras proteins must localize to the PM (Willumsen et al. 1984; Hancock et al. 1989) using a carboxy-terminal membrane anchor. This follows because Ras effectors must be recruited to the PM for activation. A Ras PM anchor consists of two components. The first anchor component, common to all Ras isoforms, is a carboxy-terminal $S$-farnesyl cysteine carboxylmethyl ester, which is generated by a sequential set of irreversible posttranslational modifications of a conserved carboxy-terminal
CAAX motif present in the nascent Ras protein (Fig. 1). The modifications are carried out by farnesyl protein transferase in the cytosol, and two endoplasmic reticulum (ER)-localized enzymes, the Rcel protease and isoprenyl-carboxymethyl transferase (Reiss et al. 1990; Dai et al. 1998; Bergo et al. 2000, 2001, 2002). The second anchor component comprises monopalmitoylation of N-Ras, duopalmitoylation of H-Ras, and a polybasic domain (PBD) of six contiguous lysines in $\mathrm{K}-\mathrm{Ras} 4 \mathrm{~B}$, the more prevalent splice form of K-Ras, hereafter called just K-Ras (Casey et al. 1989; Hancock et al. 1989, 1990). Palmitoylation is accomplished by ER- and Golgi-localized palmitoyltransferases. Of note, this type of bipartite membrane anchor is found in many other Ras superfamily proteins (Hancock et al. 1990; Michaelson et al. 2001). After anchor assembly is completed on the cytosolic surface of the ER, H-Ras, and N-Ras access the PM via the exocytic pathway through the Golgi (Choy et al. 1999; Apolloni et al. 2000), whereas K-Ras
A
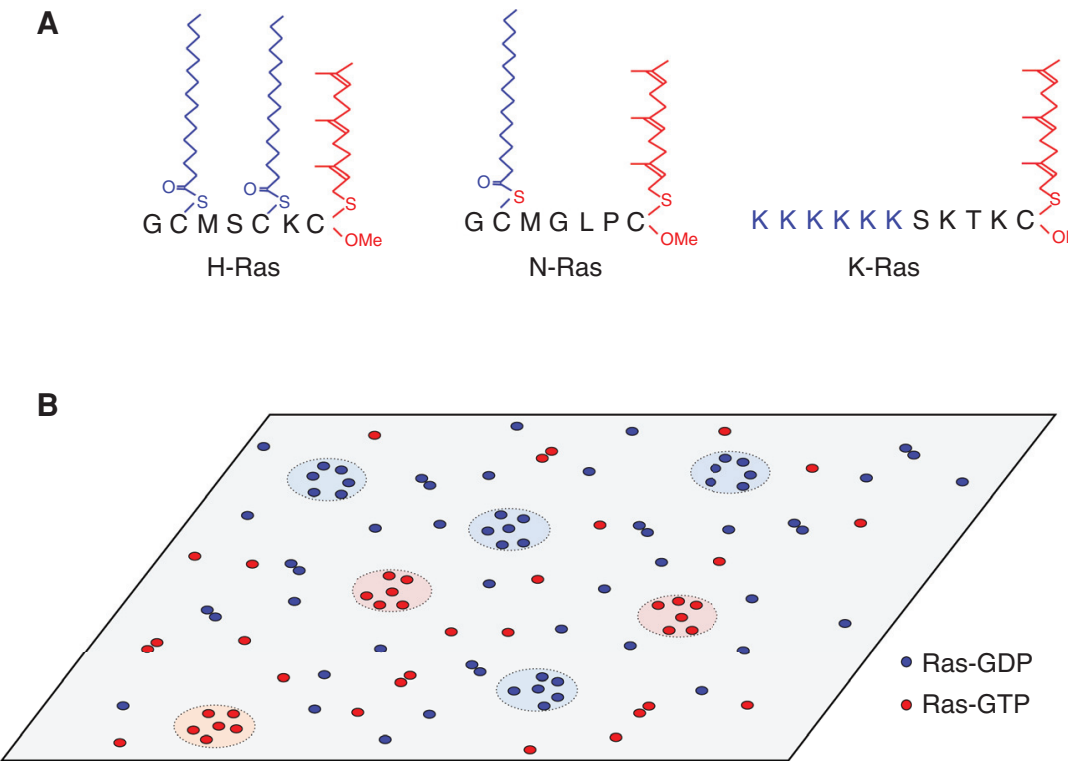

Figure 1. Ras targeting and spatial organization on the plasma membrane (PM). (A) Ras minimal membrane anchors. All Ras anchors have a farnesyl group, whereas H-Ras has two additional palmitoyl chains and N-Ras has one additional palmitoyl chain. K-Ras has a contiguous hexalysine polybasic domain (PBD). (B) Ras isoforms spatially segregate into nonoverlapping nanoclusters on cell PM. Guanine nucleotide binding states also drive further spatial separation for each isoform. Each Ras nanocluster is $\sim 18 \mathrm{~nm}$ in diameter and contains about five to six Ras molecules. $~ 56 \%$ of Ras proteins are found as monomers, whereas the remaining $44 \%$ form higherorder oligomers and nanoclusters. 
is delivered to the PM bypassing the Golgi via the recycling endosome (RE) (Choy et al. 1999; Apolloni et al. 2000; Schmick et al. 2014).

After initial delivery to the PM, each Ras protein must be actively maintained on the PM to preserve the fidelity of PM targeting and prevent entropic equilibration across all cellular membranes. To this end, $\mathrm{H}$ - and $\mathrm{N}$-Ras undergo cycles of depalmitoylation, by as-yet poorly characterized thioesterases after internalization on the cytosolic surface of endosomes, followed by repalmitoylation in the ER and Golgi for vesicular transport back to the PM (Goodwin et al. 2005; Rocks et al. 2005). Several putative chaperones that bind nonpalmitoylated $\mathrm{H}$ - and $\mathrm{N}$ Ras have been identified, including VPS35 and $\mathrm{PDE} \delta$, which facilitate cytosolic diffusion of prenylated, nonpalmitoylated Ras and contribute to engagement with membrane-bound palmitoyl transferases (Schmick et al. 2014; Zhou et al. 2016). Similarly, K-Ras is released from endosomes immediately after internalization, caused by the loss of anionic lipid asymmetry, and is captured by the chaperone protein $\mathrm{PDE} \delta$. The $\mathrm{K}$-Ras PDE $\delta$ complex is, in turn, dissociated by binding the small GTPase Arl2 in the spatial vicinity of the RE, allowing $\mathrm{K}$-Ras to bind to the cytosolic surface of RE vesicles for forward transport back to the PM (Chandra et al. 2012; Zimmermann et al. 2013; Schmick et al. 2014).

\section{SPATIAL ORGANIZATION ON THE PM: DIMERS AND NANOCLUSTERS}

The major Ras signaling platform, the PM, is a complex, dynamic structure with heterogeneity on many length and time scales (Mayor and Rao 2004; Simons and Vaz 2004; Kusumi et al. 2005; Hancock 2006; Lingwood et al. 2009; Prior and Hancock 2012). The origins of this heterogeneity include several thousand species of lipid, compartmentalization by a submembrane actin cytoskeleton, and the transient lateral assembly of different subsets of lipids driven by protein-lipid and lipid-lipid interactions (Mayor and Rao 2004; Simons and Vaz 2004; Kusumi et al. 2005; Hancock 2006; Lingwood et al. 2009; Prior and Hancock 2012). On the PM, Ras proteins diffuse laterally as monomers and dimers and assemble into higher-order oligomers that lead to the formation of nanoclusters (Prior et al. 2003; Plowman et al. 2005; Nan et al. 2013; Lin et al. 2014; Muratcioglu et al. 2015; Zhou and Hancock 2015; Jang et al. 2016; Prakash et al. 2016). A nanocluster contains about six Ras proteins, has a radius of $\sim 9 \mathrm{~nm}$ and a lifetime of $<1 \mathrm{~s}$ (Prior et al.2003; Murakoshi et al. 2004; Hancock and Parton 2005; Plowman et al. 2005; Abankwa et al. 2010; Zhou et al. 2014; Zhou and Hancock 2015). H-, N-, and K-Ras proteins assemble into spatially nonoverlapping nanoclusters with further lateral segregation between nanoclusters of GTP-loaded and GDP-loaded Ras (Prior et al. 2003; Gorfe et al. 2007b; Abankwa et al. 2008, 2010; Weise et al. 2011; Zhou et al. 2014; Prakash et al. 2016). Ras-GTP nanoclusters act as signaling platforms for effector binding and signal transmission and are therefore essential to Ras signaling (Plowman et al. 2005, 2008; Tian et al. 2007; Nan et al. 2013; Zhou and Hancock 2015) as will be discussed later.

The spatial distribution of Ras on the cell PM has been extensively quantified using electron microscopy (EM) combined with spatial analysis. EM estimates of the relative distributions of fractions, coupled with earlier single-fluorophore video tracking (SFVT) experiments, suggest that $\sim 50 \%$ of Ras proteins diffuse as monomers, with the remainder present as mobile dimers and immobile nanoclusters. This is a nonequilibrium state in that the fraction of a Ras protein population assembling into nanoclusters is constant over a multilog range of expression levels; that is, the clustered fraction is the same at endogenous levels of expression as well as ectopic expression levels reaching 20x over endogenous (Plowman et al. 2005). Interestingly, glycophosphatidylinositol (GPI)-anchored proteins show an identical nonequilibrium distribution (Mayor and Rao 2004; Goswami et al. 2008; Gowrishankar et al. 2012; Raghupathy et al. 2015). These and other considerations suggest that an intrinsic property of lipid-anchored proteins on a lipid bilayer may be a propensity to form clusters. Indeed, the clustering phenomenon is seen when multiple anchors are simulated on a bilayer using molecular dynamics (MD) and on supported bilayers (Weise et al. 
Y. Zhou et al.

2011; Li and Gorfe 2013; Lin et al. 2015). Our current understanding of the basic molecular mechanism revolves around oligomerization of the G-domain, the role of the anchor and its interactions with the lipid bilayer, and how the G-domain can modulate the behavior of the anchor (Fig. 2). These are interrelated but will be initially considered here as individual determinants. Other contributing factors that will not be considered further are thermodynamic fluctuations in lipid diffusion that result from actin turnover and trundling in the submembrane actin cytoskeleton (Goswami et al. 2008; Gowrishankar et al. 2012), which have been shown theoretically and experimentally to play a role in maintaining the nonequilibrium clustered distribution of GPI-anchored proteins and which may also therefore contribute to maintaining the similar nonequilibrium clustered distribution of Ras proteins. Connectivity between the inner and outer leaflets mediated by long-chain PtdSer and actin interactions contributes to nanoclustering of outer leaflet GPI-anchored proteins (Raghupathy et al. 2015), although relevance here to Ras is unclear.

Much recent work has focused on characterizing K-Ras dimers and putative interaction surfaces that may be relevant to PM-bound K-Ras. Dimers have been observed using super-resolution microscopy, dimers, and nanoclusters by EM of intact PM, and the existence of diverse higher-order oligomers inferred by SFVT $(\mathrm{Mu}-$ rakoshi et al. 2004; Nan et al. 2015; SpencerSmith et al. 2017). Using a combination of computational and experimental techniques, we and others recently identified two G-domain surfaces involved in the assembly of K-Ras monomers into multiple types of dimer (Muratcioglu et al. 2015; Prakash et al. 2016). The surfaces are low affinity and are stable only when the Ras protein is attached to the membrane bilayer. Both surfaces are stabilized by ionic interactions
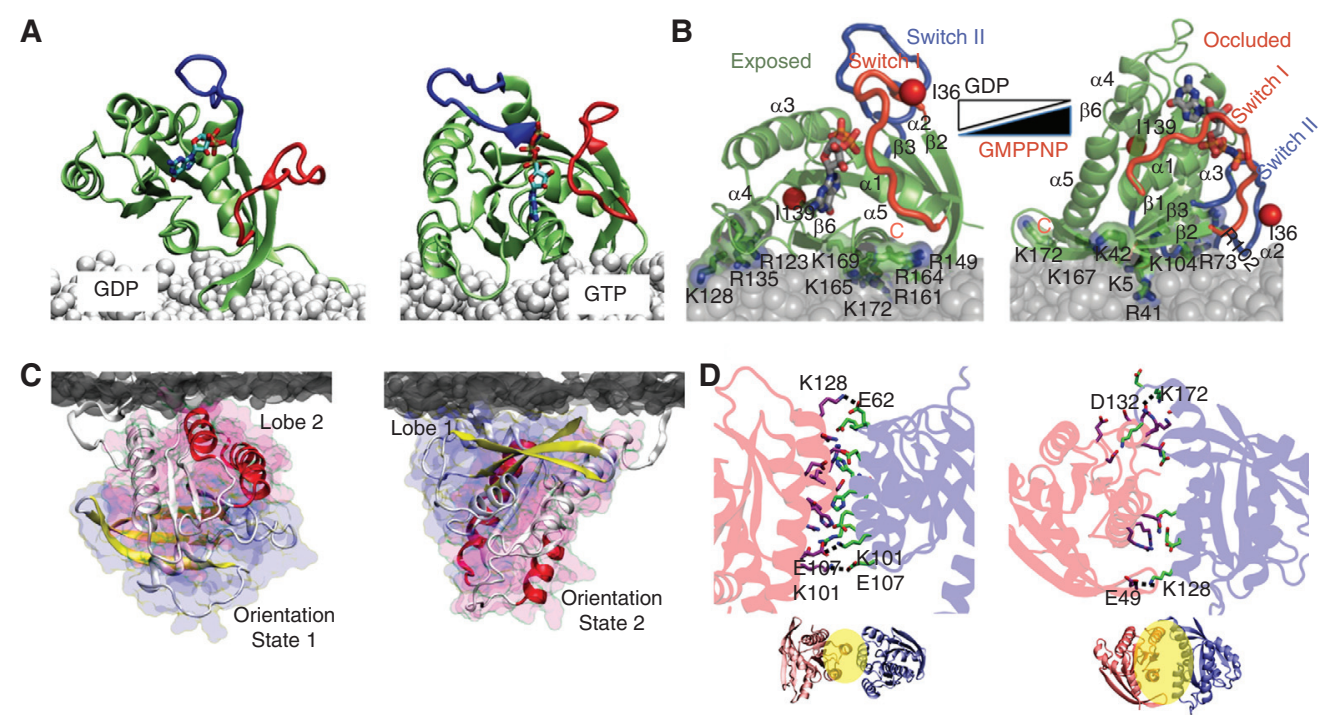

Figure 2. Ras G-domain conformational orientations drive spatial segregation on the plasma membrane. $(A) \mathrm{H}$ Ras-guanosine diphosphate (GDP) favors a G-domain orientation that is perpendicular to the membrane. Guanosine triphosphate (GTP)-bound H-Ras favors a G-domain orientation that is rotated through $100^{\circ}$ such that the helix $\alpha 4$ now interacts with lipid head groups. (B) The K-Ras G-domain is more dynamic, but samples more extensively orientate aligning parallel with the bilayer when GDP-bound and semiperpendicular when GTP-bound, with different G-domain and hypervariable region (HVR) residue hydrogen bonding to the bilayer in each case. (From Mazhab-Jafari et al. 2015; reprinted, with permission, from the National Academy of Sciences ( 2 2015.) (C) The GTP-bound K-Ras G-domain further samples two distinct conformations, OS1 and OS2 as described in the text (Prakash et al. 2016). (D) Two partially overlapping dimer surfaces that have been validated experimentally are located on helix $\alpha 3 / \alpha 4$ and helix $\alpha 4 / \alpha 5$. 
between two Ras molecules: the h3/h4 (h3= $\alpha$ helix $3 /$ h4 $=\alpha$-helix4) surface contains reciprocal K101-E107 and E107-K101 salt bridges and the h4/h5 surface contains ion pairs E98K165 and D105-K172. The role of additional $\mathrm{K}$-Ras dimerization surfaces observed in $\mathrm{MD}$ simulations remains uncertain (Muratcioglu et al. 2015; Sayyed-Ahmad et al. 2016; Prakash et al. 2017).

Taken together with the known spatial organization of K-Ras, these dimerization data lead to the hypothesis that the formation of K-Ras dimers on the PM is a prerequisite for the formation of higher-order oligomers that are, in turn, a prelude to the assembly of a nanocluster. Several lines of evidence are concordant with this hypothesis: first is the identification of multiple dimer surfaces that can be used to assemble a series of higher-order oligomers, including a pentamer (Sarkar-Banerjee et al. 2017). Of special note, the computed diameter of this pentameric structure matches exactly the lower bound diameter of a K-Ras nanocluster visualized previously by EM (Plowman et al. 2005; Sarkar-Banerjee et al. 2017). Second, if dimerization is indeed an obligate step in nanocluster assembly then perturbing dimer formation should in turn affect nanocluster formation. This has been shown experimentally in two different studies. Two sets of ionic interactions that stabilize the h3/h4 dimer or the h4/h5 dimer can be abrogated by replacing one of the partners with an amino acid of the opposite charge. Such mutations, K101E and E107K, significantly reduce K-Ras nanoclustering, whereas nanoclustering is unaffected in a double mutant K101E + E107K, in which the opposed charges are simply swapped (Prakash et al. 2017). Similarly, charge reversal mutagenesis to perturb the h4/h5 surface (E98K/D105K) impairs nanoclustering, whereas swapping the charges so that salt bridge formation is still possible (E98K/D105K + K165E/K127D) has no effect on nanoclustering (Prakash et al. 2017). Concordantly, ectopic expression of a novel monobody that targets the h3/h4 interface profoundly inhibits K-Ras dimerization, K-Ras nanoclustering, and oncogenic K-Ras signal transmission (Spencer-Smith et al. 2017). Conversely, enhancing dimerization enhances nanoclustering, for example, introducing two cysteine mutations at K101 and E107 allows the opposed cysteine side chains to form a disulfide bond, which stabilizes the h3/h4 dimer and increases K-Ras nanocluster formation (Prakash et al. 2017). Similarly, K-Ras ${ }^{\mathrm{G} 12 \mathrm{~V}}$ dimerization can be enhanced extrinsically by treating cells with BRAF kinase inhibitors to generate stable BRAF-CRAF dimers (Heidorn et al. 2010; Poulikakos et al. 2010), or by ectopically expressing a Ras-binding domain (RBD), RBDRBD dimer (Cho et al. 2012b). In both cases, the bipartite RBDs enhance K-Ras ${ }^{\mathrm{G} 12 \mathrm{~V}}$ dimerization and concomitantly enhance K-Ras nanoclustering (Cho et al. 2012b). Artificially induced dimerization of mCherry-K-Ras ${ }^{\mathrm{G} 12 \mathrm{~V}}$ also leads to significant activation of the mitogen-activated protein kinase (MAPK) signaling pathway, concordant with enhanced dimerization driving enhanced nanoclustering and, hence, increased signal output (Nan et al. 2015).

Dimers of $\mathrm{H}$-Ras and N-Ras have also been observed in MD simulations and verified experimentally (Guldenhaupt et al. 2012; Lin et al. 2014). The dimerization surfaces identified are not exactly congruous with those of K-Ras. Experiments using time-resolved fluorescence anisotropy, fluorescence correlation spectroscopy, and single-particle tracking of lipidated $\mathrm{H}$-Ras on a supported lipid bilayer (Lin et al. 2014) showed that alanine mutation of Tyr64 (Y64A) significantly increases $\mathrm{H}$-Ras lateral diffusion by eliminating dimers (Lin et al. 2014). A dimerization interface has been identified comprising helix $\alpha 5$ and loops $\beta 2 / 3$ region of $\mathrm{N}$-Ras (Guldenhaupt et al. 2012). Fluorescence lifetime measurements show that N-Ras forms extensive dimers when attached to a supported bilayer but not in solution (Guldenhaupt et al. 2012). MD simulations further propose that a dimer interface exists in the region of helices $\alpha 4, \alpha 5$ and $\beta 4$, $\alpha 5$ (Guldenhaupt et al. 2012). Key residues include intermolecular salt bridges between R161 and D154 of helix $\alpha 5$ on one N-Ras molecule with K135 in helix $\alpha 4$ and E49 in loop $\beta 2 / \beta 3$ on the adjacent $\mathrm{N}$-Ras molecule. Intermolecular salt bridges between D47 (loops $\beta 2 / \beta 3$ ) and R161/R164 of helix $\alpha 5$ on the adjacent N-Ras molecule are also prominent. There is a general 
Y. Zhou et al.

consensus among these and other studies that dimerization is restricted to membrane-bound Ras molecules because the binding affinities are too low to be detectable in solution (Dementiev 2012; Guldenhaupt et al. 2012; Lin et al. 2014; Muratcioglu et al. 2015; Nan et al. 2015; Prakash et al. 2016). Taken together, these studies also suggest that heterodimerization between different Ras isoforms would be unlikely and that homodimerization is strongly favored. A recent study examined this directly and verified that, at least for K-Ras and H-Ras, the respective dimers are isoform specific (Jang et al. 2016). This is an important result because if dimers are the building blocks of higher-order oligomers and nanoclusters, then fidelity of dimer assembly will, in turn, lead to fidelity of nanocluster assembly and provide a molecular basis for isoform-specific lateral segregation that is observed experimentally.

Complementary MD simulations and fluorescence lifetime imaging-fluorescence resonance energy transfer (FLIM-FRET) experiments have revealed that the likely basis of guanine nucleotide-dependent lateral segregation, whereby the GTP-bound and GDP-bound forms of each Ras isoform assemble into spatially nonoverlapping nanoclusters, involves conformational orientations of the G-domain with respect to the PM. For example, the H-Ras G-domain operates like a balance. In the GDP-bound state, most helices of the G-domain orient parallel to the membrane, with only the hypervariable region (HVR) and carboxy-terminal anchor engaging the membrane (Abankwa et al. 2008, 2010). This conformation allows extensive interaction of R169 and K170 with charged lipids in the membrane and the dual palmitoyl chains fully extended into the bilayer. This anchor structure can readily account for the segregation of H-Ras-GDP to ordered cholesterol-enriched domains (Prior et al. 2003; Abankwa et al. 2008, 2010). GTP binding leads to conformational reorientation and new salt bridges between D47/ E49 in the $\beta 2-\beta 3$ loops and R161/R164 in helix $\alpha 5$ (Abankwa et al. 2008). The G-domain, in turn, swings $\sim 100^{\circ}$, resulting in extensive interaction of R128 and R135 in helix $\alpha 4$ with polar lipids in the membrane and with R169 and
K170 being disengaged from the membrane (Abankwa et al. 2008, 2010). The dual palmitoyl chains also become disordered again, accounting for the segregation of H-Ras-GTP away from cholesterol-rich domains (Gorfe et al. 2007a,b; Abankwa et al. 2008, 2010).

The behavior of the K-Ras G-domain differs from that of H-Ras. Liquid nuclear magnetic resonance (NMR) analyses of K-Ras anchored to lipid nanodiscs (Mazhab-Jafari et al. 2015) and infrared spectroscopy of K-Ras on synthetic model membranes (Weise et al. 2011; Kapoor et al. 2012) show that the G-domain helices of GDP-bound K-Ras align parallel to the membrane, with the surface of $\beta 1$ and $\beta 6$, helices $\alpha 4$ and $\alpha 5$, as well as $\beta 2-\beta 3$ loops contacting acidic lipids in the bilayer. GTP loading leads to a semiperpendicular alignment of the G-domain with the membrane, with $\beta 1-3$ and helices $\alpha 2$ and $\alpha 3$ now in contact. The same alignment was predicted in all-atom MD simulations. The computational study, however, showed that KRas-GTP actually samples several distinct orientation states (OSs). A combination of MD simulations, EM-univariate spatial mapping, and FLIM-FRET revealed that K-Ras-GTP shifts between two predominant OSs (Prakash et al. 2016): OS1 presents helices $\alpha 3 / \alpha 4$ to the membrane while OS2 uses $\beta 1-3$ and helix $\alpha 2$. OS1 and OS2 are mutually exclusive in terms of interacting with the membrane and use R102, which is persistently attached to the membrane, as a hinge during switching. Detailed structures of the K-Ras minimal anchor under each nucleotide-bound state have not been reported, but the different lipid-sorting capacities discussed in the following section would suggest that they are indeed different

\section{LIPID SORTING AND LIPID COMPOSITION OF RAS NANOCLUSTERS}

Early studies showed that the cholesterol content of the PM was differentially important for $\mathrm{H}$-Ras but not K-Ras signaling and subsequently that the integrity of H-Ras-GDP and N-Ras-GTP clusters, but not K-Ras-GTP or KRas-GDP clusters, was cholesterol dependent (Roy et al. 1999, 2005; Prior et al. 2001, 2003; 
Ariotti et al. 2014). Such results suggested that each Ras nanocluster may have a different lipid composition, likely reflecting different lipidbinding preferences of the individual anchors that when, aggregated in a cluster, would in turn sort or assemble different cohorts of PM lipids. In turn, different lipid compositions of $\mathrm{H}-, \mathrm{N}-$, and K-Ras nanoclusters could account for signaling differences among the three isoforms. Subsequent work has validated each of these basic concepts.

The lipid composition of Ras nanoclusters in intact cell PM was recently determined using EM to quantify the extent of colocalization of different Ras isoforms with a set of lipid-binding probes (Figs. 3 and 4) (Zhou et al. 2014, 2015, 2017). For example, H-Ras-GDP nanoclusters were highly enriched with $\mathrm{PIP}_{2}$, whereas those of K-Ras-GTP had a high phosphatidic acid (PA) content but minimal $\mathrm{PIP}_{2}$. All Ras nanoclusters contain PtdSer, but respond to changing PtdSer levels in distinct manners. Although enhancing or decreasing PM PtdSer content increases or decreases, respectively, K-Ras ${ }^{\mathrm{G} 12 \mathrm{~V}}$ PM localization and clustering, such changes in PM PtdSer content have no effect on H-Ras ${ }^{\text {G12V }}$ (Zhou et al. 2014, 2015), leading to the conclusion that PtdSer is a critical structural component of KRas-GTP nanoclusters, but not H-Ras-GTP nanoclusters. The K-Ras PBD is a key structural element for associating with anionic lipids in the PM (Hancock et al. 1990; McLaughlin et al. 2002). Traditionally, PBDs have been thought to operate largely as charge sensors with the number of basic residues within a PBD, determining the strength of electrostatic interaction with acidic lipids and similarly interacting more strongly with acidic lipids that have a high charge density. However, K-Ras nanoclusters are enriched with monovalent PtdSer but largely de-

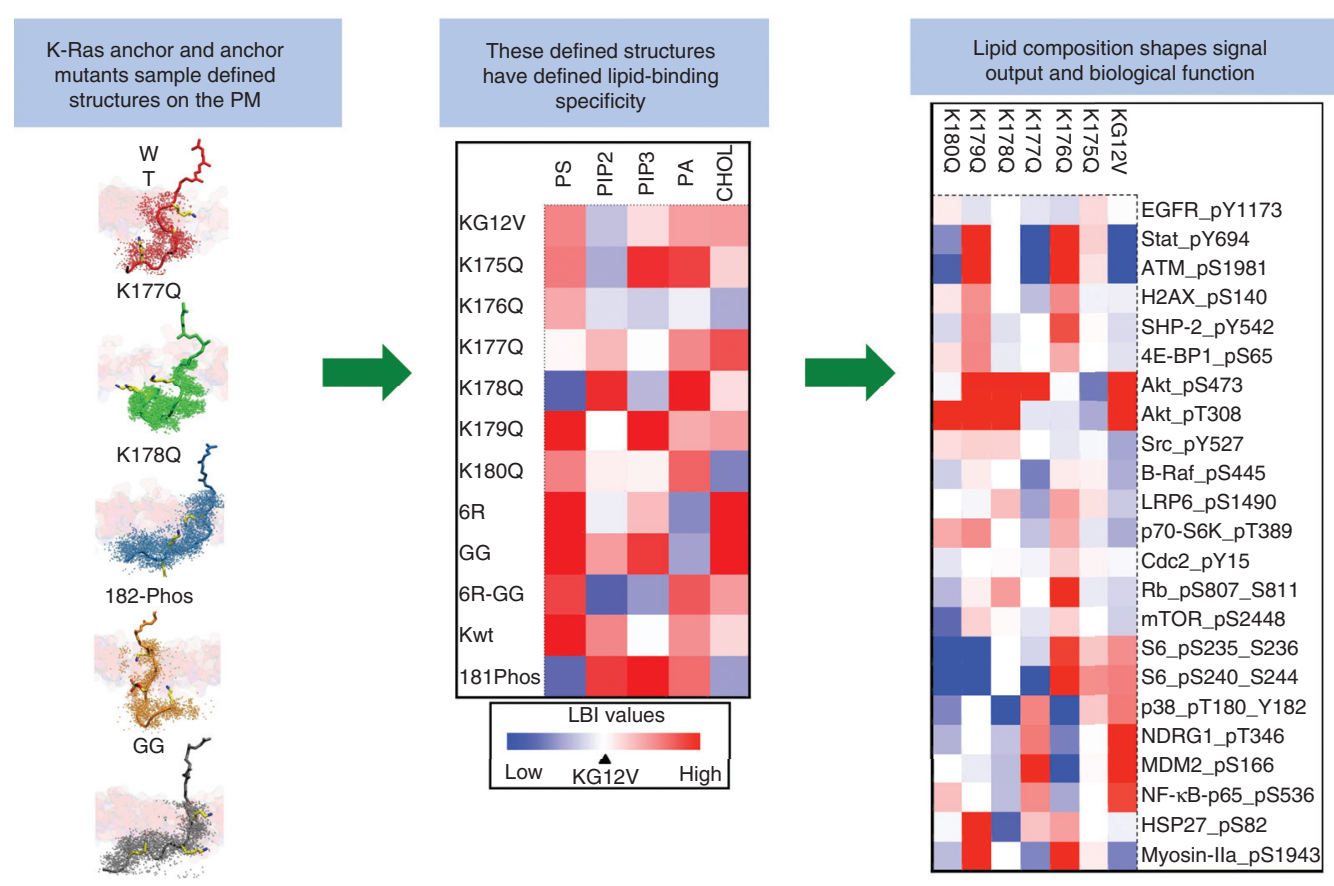

Figure 3. The K-Ras membrane anchor encodes a highly specific capacity for lipid binding. The precise amino acid sequence of the polybasic domain (PBD) and prenyl group encodes lipid-binding specificity that is realized as a defined anchor structure. Aggregation of a set of like anchors leads to assembly of nanoclusters with defined lipid compositions (shown as a heat map). The lipid composition of the resulting nanocluster shapes signal output (shown as a heat map of signaling pathway activity) (Zhou et al. 2017). LBI, Lipopolysaccharide-binding index. 
Y. Zhou et al.

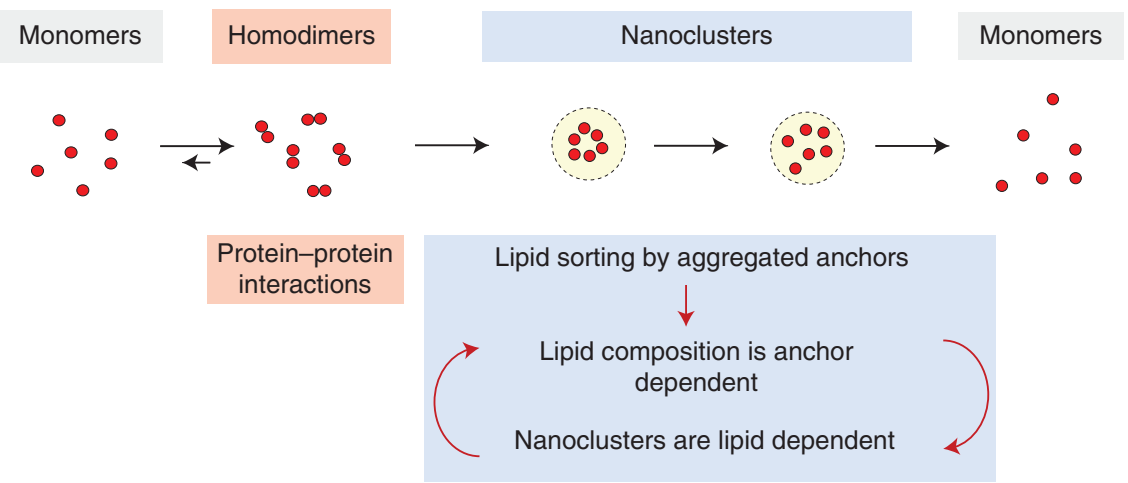

Dimerization is an obligate step for nanocluster formation

Lipid sorting defines nanocluster composition $(\Rightarrow$ isoform-specific lateral segregation)

Changes in plasma membrane lipid composition will perturb nanocluster formation

Figure 4. Putative molecular mechanism for nanocluster formation. Ras homodimer formation is an obligate step of Ras nanoclustering. Homodimers form between Ras monomers, mediated by their weak G-domain interfaces. Ras homodimers further oligomerize into nanoclusters composed of five to six proteins. Lipid sorting by the higher-order Ras oligomers generates a lipid-based nanocluster concordantly weakening G-domain interactions. The purely lipid-stabilized nanocluster then disintegrates, releasing Ras monomers.

pleted of multivalent PIP 2 (Zhou et al. 2014, 2017), suggesting that the K-Ras PBD is sensing more than just charges. Indeed, the K-Ras bipartite PBD-farnesyl anchor has now been shown to have exquisite specificity for PtdSer over anionic lipids, including $\mathrm{PIP}_{2}$, and also to selectively interact with PtdSer species that have asymmetric lipid chains comprising one fully saturated and one unsaturated acyl chain (Zhou et al. 2017). In lipid add-back experiments in PtdSer-depleted cells, K-Ras PM binding can be recovered by any PtdSer species, except PtdSer with two fully saturated chains. Strikingly, however, only asymmetric PtdSer can rescue both K-Ras PM binding and $\mathrm{K}$-Ras nanoclustering, and in turn restore the capacity of K-Ras ${ }^{\mathrm{G} 12 \mathrm{~V}}$ to recruit CRAF to the PM (Zhou et al. 2017), implying that only asymmetric PtdSer can be assembled into KRas-GTP nanoclusters.

EM bivariate colocalization analysis was then used to map lipid composition of K-Ras PBD mutants, each of which contains a single alanine point mutation at each of six different positions in the PBD. Each K-Ras PBD mutant still retains five lysines, thus equivalent to the amount of charges among all PBD mutants. Yet, each K-Ras PBD mutant possesses distinct lipid composition (Zhou et al. 2017). Of particular interest, K-RasK177Q and K-RasK178Q are depleted of PtdSer and enriched with $\mathrm{PIP}_{2}$ (Zhou et al. 2017); remarkably then, these single-point mutations are sufficient to change the lipid-binding specificity of the K-Ras anchor. Concordant with their different lipid compositions, each different PBD sequence anchor generated a different cellular signal output. Lysine and arginine each contains only one positive charge and are thus electrostatically equivalent to each other. However, replacing the lysines of the PBD with arginines fundamentally changed the lipid-binding specificity of a K-Ras anchor as does changing the farnesyl group to a geranylgeranyl group (Zhou et al. 2017). These results show that the precise amino acid sequence of the K-Ras PBD together with the prenyl group define a combinatorial code for lipid binding that extends beyond simple electrostatics. Within the code, lysine and arginine resi- 
dues are nonequivalent and prenyl chain length modifies nascent PBD lipid preferences (Zhou et al. 2017). All-atom MD simulations show that this newly discovered PBD-prenyl code embedded within the K-Ras anchor is realized by distinct dynamic tertiary structures of the anchor on the PM that govern amino acid side-chainlipid interactions (Zhou et al. 2017).

\section{POSSIBLE MOLECULAR MODEL FOR NANOCLUSTERING}

Collecting together the data and ideas described above, we can generate a working model for nanocluster assembly. We propose that G-domain-mediated Ras homodimerization is an obligate step for Ras nanocluster formation. Freely diffusing Ras homodimers assemble as a result of molecular collisions into higher-order oligomers up to and including pentamers. A key consequence of the assembly of these higher-order structures is an aggregation of a set of identical carboxy-terminal anchors with defined lipidbinding specificity that can sort or retain specific cohorts of PM lipids. Each nanocluster therefore acquires a defined, nonrandom assembly of lipids. We speculate that as lipid composition of the nanocluster is established, protein-protein interactions are destabilized, leaving the nanocluster as a lipid-based assembly of Ras monomers. This is an intrinsically unstable entity with a finite lifetime that disassembles as lipids diffuse away, releasing a set of freely diffusing Ras monomers.

\section{SIGNAL PROCESSING AND EMERGENT SYSTEM PROPERTIES OF THE RAS NANOCLUSTER SYSTEM}

RAF, MEK, and ERK are recruited and activated in Ras nanoclusters not by freely diffusing Ras monomers (Tian et al. 2007; Plowman et al. 2008). This spatiotemporal restriction of MAPK cascade activation results in key emergent properties, including high-fidelity and low-noise signal transmission within the RasMAPK signaling circuit (Tian et al. 2007; Harding and Hancock 2008b; Kholodenko et al. 2010). In brief, the fixed nanoclustered fraction $(\phi)$ at all Ras expression levels results in a linear relationship between the amount of Ras-GTP on the PM, generated, for example, in response to epidermal growth factor receptor (EGFR) activation and the number of Ras-GTP nanoclusters assembled (Fig. 5). Each nanocluster then operates as a transient digital switch in activating MAPK, generating a fixed quantal output of ERKpp before the nanocluster disassembles and terminates MAPK activation (Harding et al. 2005; Tian et al. 2007, 2010; Harding and Hancock 2008a,b; Inder and Hancock 2008; Inder et al. 2008; Kholodenko et al. 2010). The whole system operates as an analog-digitalanalog converter, in which the assembly of nanoclusters digitizes the incoming analog signal (acting as an analog-to-digital converter [ADC]). The cytosolic summed output of all the ERKpp generated by the nanoclusters then regenerates the analog input signal (acting as a digital-to-analog converter [DAC]). These features of K-Ras nanoclusters allow cells to use digital biochemistry to build high-fidelity analog signaling circuits (Tian et al. 2007; Harding and Hancock 2008b; Kholodenko et al. 2010). The dynamics of nanocluster assembly and turnover are thus critical for Ras function (Plowman et al. 2005; Tian et al. 2007; Kholodenko et al. 2010) such that the nanoclustered fraction $(\phi)$ becomes a critical system parameter that can set signal gain. Increasing $\phi$ results in increases in signal gain, whereby there is increased MAPK activation per unit of Ras-GTP, whereas reducing $\phi$ has the opposite effect; at the limit, completely blocking nanocluster formation abrogates Ras signaling even if Ras is GTP loaded and PM localized.

In intact cells, $\phi$ can be regulated by multiple mechanisms. Extensive early studies implicated galectin-1 and galectin-3 as H-Ras-GTP and K-Ras-GTP nanocluster scaffolds, respectively (Belanis et al. 2008; Shalom-Feuerstein et al. 2008). Increasing the cytosolic concentration of these galectins enhanced H-Ras and K-Ras nanoclustering and MAPK signaling concordantly. Intriguingly, cytosolic levels of nucleophosmin and nucleolin also enhance K-Ras clustering (Inder et al. 2009). Caveolae remotely control Ras nanoclustering in part by sequestering PtdSer (Ariotti et al. 2014; Zhou et al. 2014). Disruption of caveolae by expressing 
Y. Zhou et al.

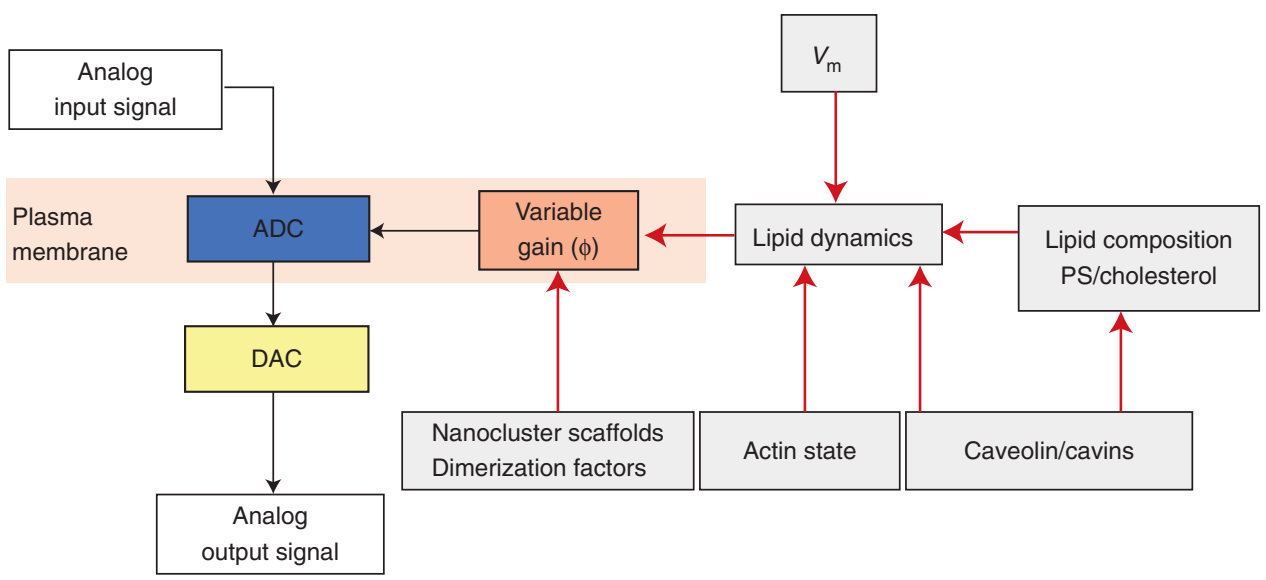

$A D C=$ analog-to-digital converter (formation of K-Ras-GTP nanoclusters)

$\mathrm{DAC}=$ digital-to-analog converter (sum of nanocluster ERKpp outputs)

$\phi=\mathrm{K}-$ Ras clustered fraction

Figure 5. Multiple plasma membrane (PM) mechanisms regulate the gain in Ras signaling circuits. Ras nanoclusters set up the PM as an analog-digital-analog converter for high-fidelity mitogen-activated protein kinase (MAPK) signal transmission. The Ras clustered fraction $(\phi)$ is a key system parameter that sets the gain in the signaling circuit and that can, in turn, be controlled by multiple mechanisms as indicated on the diagram. Of particular interest are mechanisms that use PM lipid dynamics or effective lipid composition to regulate $\phi$, including $V_{\mathrm{m}}$ (transmembrane potential). In this context, the PM can operate as a high-level controller of signal transmission. PS, Phosphatidylserine.

dominant-negative caveolin mutants, or knockdown of caveolin or cavin, alters PM PtdSer and cholesterol content and distribution to enhance K-Ras-GTP clustering, but abolish GTP-dependent lateral segregation of $\mathrm{H}$-Ras (Ariotti et al. 2014; Zhou et al. 2014). PM depolarization also induces nanoscale reorganization PtdSer and $\mathrm{PIP}_{2}$ but not other anionic phospholipids (Zhou et al. 2015). The reorganization of PtdSer, in turn, enhances K-Ras nanoclustering, which robustly amplifies K-Ras-dependent MAPK signaling. Conversely, PM repolarization disrupts $\mathrm{K}$-Ras nanoclustering and inhibits MAPK signaling. Therefore, by responding to voltage-induced changes in PtdSer spatiotemporal dynamics, K-Ras nanoclusters set up the PM as a biological field-effect transistor, allowing membrane potential to control the gain in mitogenic signaling circuits. Together, these results show that $\phi$ is an important parameter, subject to multiple levels of control that directly control the strength of Ras signal transduction.

The important role of PtdSer in regulating KRas spatiotemporal organization on cell PM and signaling is further shown by the pharmacology of two K-Ras inhibitors: fendiline and staurosporine (STS). Both of these compounds perturb sphingomyelin metabolism, fendiline, by inhibiting acid sphingomyelinase, and STS by inhibiting the ORMDL complex that inhibits the first step in sphingomyelin biosynthesis (Cho et al. 2012a, 2015; van der Hoeven et al. 2013; Maekawa et al. 2016). Both compounds cause sphingomyelin loading of the endolysomal system, leading to aberrant lipid trafficking and loss of PtdSer and cholesterol from the PM. Both compounds in consequence therefore potently suppress K-Ras signaling, arguing that pharmacological targeting of PM lipid content may have some merit as an approach to block oncogenic $\mathrm{K}$-Ras function.

\section{CONCLUDING REMARKS}

Ras proteins spatially segregate into distinct nanoclusters on the PM in an isoform- and guanine nucleotide-dependent manner. Nanocluster formation is essential to Ras-dependent 
signal transmission because these transient lipid-dependent assemblies operate as platforms for effector binding and activation. The molecular mechanisms that lead to nanocluster formation are becoming clearer and involve contributions from protein-protein interactions, largely confined to the G-domain, and protein-lipid interactions involving both the G-domain and the carboxy-terminal lipid anchor. Other small GTPases also share similar G-domain structures and analogous bipartite lipid anchors, and we speculate that these proteins, including Rho, Rac, and CDC42, will all undergo lateral segregation into distinct nanoclusters with specific lipid compositions, using the same basic molecular and biophysical mechanisms as Ras. These mechanisms can, on the one hand, be used by the cell to tune Ras signal transmission, and on the other hand may offer novel therapeutic approaches to block oncogenic Ras function.

\section{ACKNOWLEDGMENTS}

Work in the authors' laboratories is supported by the Cancer Research and Prevention Institute of Texas (CPRIT: RP170233 and DP150065) to J.F.H. and the National Institutes of Health (NIH: P30 DK056338) to Y.Z.

\section{REFERENCES}

Abankwa D, Hanzal-Bayer M, Ariotti N, Plowman SJ, Gorfe AA, Parton RG, McCammon JA, Hancock JF. 2008. A novel switch region regulates $\mathrm{H}$-Ras membrane orientation and signal output. EMBO J 27: 727-735.

Abankwa D, Gorfe AA, Inder K, Hancock JF. 2010. Ras membrane orientation and nanodomain localization generate isoform diversity. Proc Natl Acad Sci 107: 11301135.

Apolloni A, Prior IA, Lindsay M, Parton RG, Hancock JF. 2000. H-Ras but not K-Ras traffics to the plasma membrane through the exocytic pathway. Mol Cell Biol 20: 2475-2487.

Ariotti N, Fernández-Rojo MA, Zhou Y, Hill MM, Rodkey T, Inder K, Hancock JF, Parton RG. 2014. Caveolae regulate the nanoscale organization of the plasma membrane to remotely control Ras signaling. J Cell Biol 204: 777-792.

Belanis L, Plowman SJ, Rotblat B, Hancock JF, Kloog Y. 2008 Galectin-1 is a novel structural component and a major regulator of H-Ras nanoclusters. Mol Biol Cell 19: 14041414.
Bergo MO, Leung GK, Ambroziak P, Otto JC, Casey PJ Young SG. 2000. Targeted inactivation of the isoprenylcysteine carboxyl methyltransferase gene causes mislocalization of K-Ras in mammalian cells. J Biol Chem 23: 17605-17610.

Bergo MO, Leung GK, Ambroziak P, Otto JC, Casey PJ, Gomes AQ, Seabra MC, Young SG. 2001. Isoprenylcysteine carboxyl methyltransferase deficiency in mice. J Biol Chem 276: 5841-5845.

Bergo MO, Ambroziak P, Gregory C, George A, Otto JC, Kim E, Nagase H, Casey PJ, Balmain A, Young SG. 2002. Absence of the CAAX endoprotease Rcel: Effects on cell growth and transformation. Mol Cell Biol 22: 171181.

Casey PJ, Solski PA, Der CJ, Buss JE. 1989. p21 ras is modified by a farnesyl isoprenoid. Proc Natl Acad Sci 86: 83238327.

Chandra A, Grecco HE, Pisupati V, Perera D, Cassidy L, Skoulidis F, Ismail SA, Hedberg C, Hanzal-Bayer M, Venkitaraman AR, et al. 2012. The GDI-like solubilizing factor PDE $\delta$ sustains the spatial organization and signalling of Ras family proteins. Nat Cell Biol 14: 148-158.

Cho K-j, Park J-H, Piggott AM, Salim A, Gorfe AA, Parton RG, Capon RJ, Lacey E, Hancock JF. 2012a. Staurosporines disrupt phosphatidylserine trafficking and mislocalize Ras proteins. J Biol Chem 287: 43573-43584.

Cho KJ, Kasai RS, Park JH, Chigurupati S, Heidorn SJ, van der Hoeven D, Plowman SJ, Kusumi A, Marais R, Hancock JF. 2012b. Raf inhibitors target Ras spatiotemporal dynamics. Curr Biol 22: 945-955.

Cho KJ, van der Hoeven D, Zhou Y, Maekawa M, Ma X, Chen W, Fairn GD, Hancock JF. 2015. Inhibition of acid sphingomyelinase depletes cellular phosphatidylserine and mislocalizes K-Ras from the plasma membrane. Mol Cell Biol 36: 363-374.

Choy E, Chiu VK, Silletti J, Feoktisitov M, Morimoto T, Michaelson D, Ivanov IE, Philips MR. 1999. Endomembrane trafficking of Ras: The CAAX motif targets proteins to the ER and Golgi. Cell 98: 69-80.

Dai Q, Choy E, Chiu V, Romano J, Slivka SR, Seitz SA, Michaelis S, Philips MR. 1998. Mammalian prenylcysteine carboxyl methyltransferase is in the endoplasmic reticulum. J Biol Chem 273: 15030-15034.

Dementiev A. 2012. K-Ras4B lipoprotein synthesis: Biochemical characterization, functional properties, and dimer formation. Protein Expr Purif 84: 86-93.

Goodwin JS, Drake KR, Rogers C, Wright L, LippincottSchwartz J, Philips MR, Kenworthy AK. 2005. Depalmitoylated Ras traffics to and from the Golgi complex via a nonvesicular pathway. J Cell Biol 170: 261-272.

Gorfe AA, Babakhani A, McCammon JA. 2007a. H-Ras protein in a bilayer: Interaction and structure perturbation. J Am Chem Soc 129: 12280-12286.

Gorfe AA, Hanzal-Bayer M, Abankwa D, Hancock JF, McCammon JA. 2007b. Structure and dynamics of the full-length lipid-modified H-Ras protein in a 1,2-dimyristoylglycero-3-phosphocholine bilayer. J Med Chem 50: 674-684.

Goswami D, Gowrishankar K, Bilgrami S, Ghosh S, Raghupathy R, Chadda R, Vishwakarma R, Rao M, Mayor S. 2008. Nanoclusters of GPI-anchored proteins are formed by cortical actin-driven activity. Cell 135: 1085-1097. 
Y. Zhou et al.

Gowrishankar K, Ghosh S, Saha S, C R, Mayor S, Rao M. 2012. Active remodeling of cortical actin regulates spatiotemporal organization of cell surface molecules. Cell 149: 1353-1367.

Guldenhaupt J, Rudack T, Bachler P, Mann D, Triola G, Waldmann H, Kotting C, Gerwert K. 2012. N-Ras forms dimers at POPC membranes. Biophys J 103: 1585-1593.

Hancock JF. 2003. Ras proteins: Different signals from different locations. Nat Rev Mol Cell Biol 4: 373-384.

Hancock JF. 2006. Lipid rafts: Contentious only from simplistic standpoints. Nat Rev Mol Cell Biol 7: 456-462.

Hancock JF, Parton RG. 2005. Ras plasma membrane signalling platforms. Biochem J 389: 1-11.

Hancock JF, Magee AI, Childs JE, Marshall CJ. 1989. All ras proteins are polyisoprenylated but only some are palmitoylated. Cell 57: 1167-1177.

Hancock JF, Paterson H, Marshall CJ. 1990. A polybasic domain or palmitoylation is required in addition to the CAAX motif to localize p21 ras to the plasma membrane. Cell 63: 133-139.

Harding A, Hancock JF. 2008a. Ras nanoclusters: Combining digital and analog signaling. Cell Cycle 7: 127-134.

Harding AS, Hancock JF. 2008b. Using plasma membrane nanoclusters to build better signaling circuits. Trends Cell Biol 18: 364-371.

Harding A, Tian T, Westbury E, Frische E, Hancock JF. 2005. Subcellular localization determines MAP kinase signal output. Curr Biol 15: 869-873.

Heidorn SJ, Milagre C, Whittaker S, Nourry A, NiculescuDuvas I, Dhomen N, Hussain J, Reis-Filho JS, Springer CJ, Pritchard C, et al. 2010. Kinase-dead BRAF and oncogenic RAS cooperate to drive tumor progression through CRAF. Cell 140: 209-221.

Inder K, Hancock JF. 2008. System output of the MAPK module is spatially regulated. Commun Integr Biol 1: $178-179$.

Inder K, Harding A, Plowman SJ, Philips MR, Parton RG, Hancock JF. 2008. Activation of the MAPK module from different spatial locations generates distinct system outputs. Mol Biol Cell 19: 4776-4784.

Inder KL, Lau C, Loo D, Chaudhary N, Goodall A, Martin S, Jones A, van der Hoeven D, Parton RG, Hill MM, et al. 2009. Nucleophosmin and nucleolin regulate K-Ras plasma membrane interactions and MAPK signal transduction. J Biol Chem 284: 28410-28419.

Jang H, Muratcioglu S, Gursoy A, Keskin O, Nussinov R. 2016. Membrane-associated Ras dimers are isoform-specific: K-Ras dimers differ from H-Ras dimers. Biochem $J$ 473: 1719-1732.

Kapoor S, Weise K, Erlkamp M, Triola G, Waldmann H, Winter R. 2012. The role of G-domain orientation and nucleotide state on the Ras isoform-specific membrane interaction. Eur Biophys J 41: 801-813.

Kholodenko BN, Hancock JF, Kolch W. 2010. Signalling ballet in space and time. Nat Rev Mol Cell Biol 11: 414426.

Kusumi A, Nakada C, Ritchie K, Murase K, Suzuki K, Murakoshi H, Kasai RS, Kondo J, Fujiwara T. 2005. Paradigm shift of the plasma membrane concept from the two-dimensional continuum fluid to the partitioned fluid: High- speed single-molecule tracking of membrane molecules. Annu Rev Biophys Biomol Struct 34: 351-378.

Li Z, Gorfe AA. 2013. Deformation of a two-domain lipid bilayer due to asymmetric insertion of lipid-modified Ras peptides. Soft Matter doi: 10.1039/C3SM51388B.

Lin WC, Iversen L, Tu HL, Rhodes C, Christensen SM, Iwig JS, Hansen SD, Huang WY, Groves JT. 2014. H-Ras forms dimers on membrane surfaces via a protein-protein interface. Proc Natl Acad Sci 111: 2996-3001.

Lin X, Li Z, Gorfe AA. 2015. Reversible effects of peptide concentration and lipid composition on H-Ras lipid anchor clustering. Biophys J 109: 2467-2470.

Lingwood D, Kaiser HJ, Levental I, Simons K. 2009. Lipid rafts as functional heterogeneity in cell membranes. Biochem Soc Trans 37: 955-960.

Maekawa M, Lee M, Wei K, Ridgway ND, Fairn GD. 2016. Staurosporines decrease ORMDL proteins and enhance sphingomyelin synthesis resulting in depletion of plasmalemmal phosphatidylserine. Sci Rep 6: 35762.

Mayor S, Rao M. 2004. Rafts: Scale-dependent, active lipid organization at the cell surface. Traffic 5: 231-240.

Mazhab-Jafari MT, Marshall CB, Smith MJ, Gasmi-Seabrook GM, Stathopulos PB, Inagaki F, Kay LE, Neel BG, Ikura M. 2015. Oncogenic and RASopathy-associated KRAS mutations relieve membrane-dependent occlusion of the effector-binding site. Proc Natl Acad Sci 112: 6625-6630.

McLaughlin S, Wang J, Gambhir A, Murray D. 2002. $\mathrm{PIP}_{2}$ and proteins: Interactions, organization, and information flow. Annu Rev Biophys Biomol Struct 31: 151-175.

Michaelson D, Silletti J, Murphy G, D’Eustachio P, Rush M, Philips MR. 2001. Differential localization of Rho GTPases in live cells: Regulation by hypervariable regions and RhoGDI binding. J Cell Biol 152: 111-126.

Murakoshi H, Iino R, Kobayashi T, Fujiwara T, Ohshima C, Yoshimura A, Kusumi A. 2004. Single-molecule imaging analysis of Ras activation in living cells. Proc Natl Acad Sci 101: 7317-7322.

Muratcioglu S, Chavan TS, Freed BC, Jang H, Khavrutskii L, Freed RN, Dyba MA, Stefanisko K, Tarasov SG, Gursoy A et al. 2015. GTP-dependent K-Ras dimerization. Structure 23: 1325-1335.

Nan X, Collisson EA, Lewis S, Huang J, Tamguney TM, Liphardt JT, McCormick F, Gray JW, Chu S. 2013. Single-molecule superresolution imaging allows quantitative analysis of RAF multimer formation and signaling. Proc Natl Acad Sci 110: 18519-18524.

Nan X, Tamguney TM, Collisson EA, Lin LJ, Pitt C, Galeas J, Lewis S, Gray JW, McCormick F, Chu S. 2015. Ras-GTP dimers activate the mitogen-activated protein kinase (MAPK) pathway. Proc Natl Acad Sci 112: 7996-8001.

Plowman SJ, Muncke C, Parton RG, Hancock JF. 2005. $\mathrm{H}$-Ras, K-Ras and inner plasma membrane raft proteins operate in nanoclusters that exhibit differential dependence on the actin cytoskeleton. Proc Natl Acad Sci 102: 15500-15505.

Plowman SJ, Ariotti N, Goodall A, Parton RG, Hancock JF. 2008. Electrostatic interactions positively regulate K-Ras nanocluster formation and function. Mol Cell Biol 28: 4377-4385. 
Poulikakos PI, Zhang C, Bollag G, Shokat KM, Rosen N 2010. RAF inhibitors transactivate RAF dimers and ERK signalling in cells with wild-type BRAF. Nature 464: 427-430.

Prakash P, Zhou Y, Liang H, Hancock JF, Gorfe AA. 2016 Oncogenic K-Ras binds to an anionic membrane in two distinct orientations: A molecular dynamics analysis. $B i$ ophys J 110: 1125-1138.

Prakash P, Sayyed-Ahmad A, Cho KJ, Dolino DM, Chen W, Li H, Grant BJ, Hancock JF, Gorfe AA. 2017. Computational and biochemical characterization of two partially overlapping interfaces and multiple weak-affinity K-Ras dimers. Sci Rep 7: 40109.

Prior IA, Hancock JF. 2001. Compartmentalization of Ras proteins. J Cell Sci 114: 1603-1608.

Prior IA, Hancock JF. 2012. Ras trafficking, localization and compartmentalized signalling. Semin Cell Dev Biol 23: 145-153.

Prior IA, Harding A, Yan J, Sluimer J, Parton RG, Hancock JF. 2001. GTP-dependent segregation of H-Ras from lipid rafts is required for biological activity. Nat Cell Biol 3: 368-375.

Prior IA, Muncke C, Parton RG, Hancock JF. 2003. Direct visualization of Ras proteins in spatially distinct cell surface microdomains. J Cell Biol 160: 165-170.

Prior IA, Lewis PD, Mattos C. 2012. A comprehensive survey of Ras mutations in cancer. Cancer Res 72: 2457-2467.

Raghupathy R, Anilkumar AA, Polley A, Singh PP, Yadav M, Johnson C, Suryawanshi S, Saikam V, Sawant SD, Panda A, et al. 2015. Transbilayer lipid interactions mediate nanoclustering of lipid-anchored proteins. Cell 161: 581-594.

Reiss Y, Goldstein JL, Seabra MC, Casey PJ, Brown MS. 1990 Inhibition of purified $\mathrm{p} 21^{\text {ras }}$ farnesyl:protein transferase by Cys-AAX tetrapeptides. Cell 62: 81-88.

Rocks O, Peyker A, Kahms M, Verveer PJ, Koerner C, Lumbierres M, Kuhlmann J, Waldmann H, Wittinghofer A, Bastiaens PI. 2005. An acylation cycle regulates localization and activity of palmitoylated Ras isoforms. Science 307: 1746-1752.

Roy S, Luetterforst R, Harding A, Apolloni A, Etheridge M, Stang E, Rolls B, Hancock JF, Parton RG. 1999. Dominant-negative caveolin inhibits $\mathrm{H}$-Ras function by disrupting cholesterol-rich plasma membrane domains. Nat Cell Biol 1: 98-105.

Roy S, Plowman S, Rotblat B, Prior IA, Muncke C, Grainger S, Parton RG, Henis YI, Kloog Y, Hancock JF. 2005. Individual palmitoyl residues serve distinct roles in H-Ras trafficking, microlocalization, and signaling. Mol Cell Biol 25: 6722-6733.

Sarkar-Banerjee S, Sayyed-Ahmad A, Prakash P, Cho K-J, Waxham MN, Hancock JF, Gorfe AA. 2017. Spatiotemporal analysis of K-Ras plasma membrane interactions reveals multiple high order homo-oligomeric complexes. J Am Chem Soc 139: 13466-13475.

Sayyed-Ahmad A, Cho KJ, Hancock JF, Gorfe AA. 2016. Computational equilibrium thermodynamic and kinetic analysis of K-Ras dimerization through an effector binding surface suggests limited functional role. J Phys Chem B 120: $8547-8556$.
Schmick M, Vartak N, Papke B, Kovacevic M, Truxius DC, Rossmannek L, Bastiaens PI. 2014. KRas localizes to the plasma membrane by spatial cycles of solubilization, trapping and vesicular transport. Cell 157: 459-471.

Shalom-Feuerstein R, Plowman SJ, Rotblat B, Ariotti N, Tian T, Hancock JF, Kloog Y. 2008. K-Ras nanoclustering is subverted by overexpression of the scaffold protein galectin-3. Cancer Res 68: 6608-6616.

Simons K, Vaz WL. 2004. Model systems, lipid rafts, and cell membranes. Annu Rev Biophys Biomol Struct 33: 269295.

Spencer-Smith R, Koide A, Zhou Y, Eguchi RR, Sha F, Gajwani P, Santana D, Gupta A, Jacobs M, Herrero-Garcia E, et al. 2017. Inhibition of RAS function through targeting an allosteric regulatory site. Nat Chem Biol 13: 62-68.

Tian T, Harding A, Inder K, Plowman S, Parton RG, Hancock JF. 2007. Plasma membrane nanoswitches generate high-fidelity Ras signal transduction. Nat Cell Biol 9: 905914.

Tian T, Plowman SJ, Parton RG, Kloog Y, Hancock JF. 2010. Mathematical modeling of K-Ras nanocluster formation on the plasma membrane. Biophys J 99: 534-543.

van der Hoeven D, Cho KJ, Ma X, Chigurupati S, Parton RG, Hancock JF. 2013. Fendiline inhibits K-Ras plasma membrane localization and blocks K-Ras signal transmission. Mol Cell Biol 33: 237-251.

Weise K, Kapoor S, Denter C, Nikolaus J, Opitz N, Koch S, Triola G, Herrmann A, Waldmann H, Winter R. 2011. Membrane-mediated induction and sorting of K-Ras microdomain signaling platforms. J Am Chem Soc 133: 880887.

Willumsen BM, Christensen A, Hubbert NL, Papageorge AG, Lowy DR. 1984. The p21 ras C-terminus is required for transformation and membrane association. Nature 310: 583-586.

Zhou Y, Hancock JF. 2015. Ras nanoclusters: Versatile lipidbased signaling platforms. Biochim Biophys Acta 1853: 841-849.

Zhou Y, Liang H, Rodkey T, Ariotti N, Parton RG, Hancock JF. 2014. Signal integration by lipid-mediated spatial cross talk between Ras nanoclusters. Mol Cell Biol 34: 862-876.

Zhou Y, Wong CO, Cho KJ, van der Hoeven D, Liang H, Thakur DP, Luo J, Babic M, Zinsmaier KE, Zhu MX, et al. 2015. Membrane potential modulates plasma membrane phospholipid dynamics and K-Ras signaling. Science 349: 873-876.

Zhou M, Wiener H, Su W, Zhou Y, Liot C, Ahearn I, Hancock JF, Philips MR. 2016. VPS35 binds farnesylated NRas in the cytosol to regulate N-Ras trafficking. J Cell Biol 214: 445-458.

Zhou Y, Prakash P, Liang H, Cho KJ, Gorfe AA, Hancock JF. 2017. Lipid-sorting specificity encoded in K-Ras membrane anchor regulates signal output. Cell 168: 239-251.

Zimmermann G, Papke B, Ismail S, Vartak N, Chandra A, Hoffmann M, Hahn SA, Triola G, Wittinghofer A, Bastiaens PI, et al. 2013. Small molecule inhibition of the KRAS-PDE $\delta$ interaction impairs oncogenic KRAS signalling. Nature 497: 638-642. 


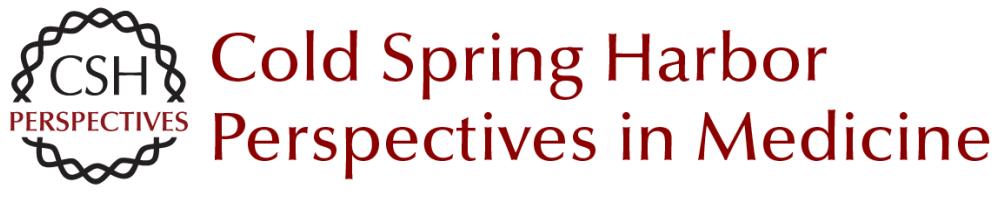

\section{Ras and the Plasma Membrane: A Complicated Relationship}

Yong Zhou, Priyanka Prakash, Alemayehu A. Gorfe and John F. Hancock

Cold Spring Harb Perspect Med 2018; doi: 10.1101/cshperspect.a031831 originally published online December 11, 2017

\section{Subject Collection Ras and Cancer in the 21st Century}

Targeting Ras with Macromolecules Dehua Pei, Kuangyu Chen and Hui Liao

Ras-Specific GTPase-Activating Proteins-Structures, Mechanisms, and Interactions Klaus Scheffzek and Giridhar Shivalingaiah

Ras-Mediated Activation of the Raf Family Kinases Elizabeth M. Terrell and Deborah K. Morrison

Posttranslational Modifications of RAS Proteins Ian Ahearn, Mo Zhou and Mark R. Philips

Kras in Organoids Derek Cheng and David Tuveson

KRAS: The Critical Driver and Therapeutic Target for Pancreatic Cancer Andrew M. Waters and Channing J. Der

The K-Ras, N-Ras, and H-Ras Isoforms: Unique Conformational Preferences and Implications for Targeting Oncogenic Mutants Jillian A. Parker and Carla Mattos

PI3K: A Crucial Piece in the RAS Signaling Puzzle Agata Adelajda Krygowska and Esther Castellano
MRAS: A Close but Understudied Member of the RAS Family Lucy C. Young and Pablo Rodriguez-Viciana

The Interdependent Activation of

Son-of-Sevenless and Ras Pradeep Bandaru, Yasushi Kondo and John Kuriyan

Targeting the MAPK Pathway in RAS Mutant Cancers

Sarah G. Hymowitz and Shiva Malek

Ras and the Plasma Membrane: A Complicated

Relationship

Yong Zhou, Priyanka Prakash, Alemayehu A. Gorfe, et al.

Kras and Tumor Immunity: Friend or Foe? Jane Cullis, Shipra Das and Dafna Bar-Sagi

Synthetic Lethal Vulnerabilities in KRAS-Mutant Cancers Andrew J. Aguirre and William C. Hahn

Efforts to Develop KRAS Inhibitors Matthew Holderfield

Genetically Engineered Mouse Models of K-Ras-Driven Lung and Pancreatic Tumors: Validation of Therapeutic Targets Matthias Drosten, Carmen Guerra and Mariano Barbacid

For additional articles in this collection, see http://perspectivesinmedicine.cshlp.org/cgi/collection/ 